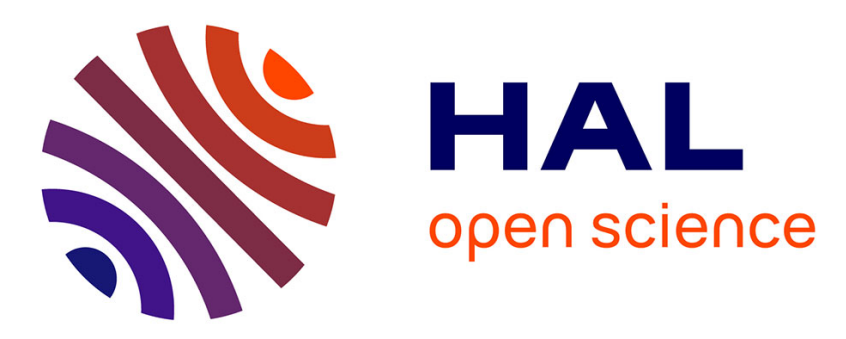

\title{
Extraction of Silicone Uncrosslinked Chains at Air-Water-Polydimethylsiloxane Triple Lines
}

Aurélie Hourlier-Fargette, Julien Dervaux, Arnaud Antkowiak, Sebastien

Neukirch

\section{- To cite this version:}

Aurélie Hourlier-Fargette, Julien Dervaux, Arnaud Antkowiak, Sebastien Neukirch. Extraction of Silicone Uncrosslinked Chains at Air-Water-Polydimethylsiloxane Triple Lines. Langmuir, 2018, 34 (41), pp.12244-12250. hal-01944633

\section{HAL Id: hal-01944633 \\ https: / hal.sorbonne-universite.fr/hal-01944633}

Submitted on 4 Dec 2018

HAL is a multi-disciplinary open access archive for the deposit and dissemination of scientific research documents, whether they are published or not. The documents may come from teaching and research institutions in France or abroad, or from public or private research centers.
L'archive ouverte pluridisciplinaire HAL, est destinée au dépôt et à la diffusion de documents scientifiques de niveau recherche, publiés ou non, émanant des établissements d'enseignement et de recherche français ou étrangers, des laboratoires publics ou privés. 


\title{
Extraction of silicone uncrosslinked chains at air-water-PDMS triple lines
}

\author{
Aurélie Hourlier-Fargette, ${ }^{*, \dagger, \ddagger}$ Julien Dervaux, Arnaud Antkowiak, ${ }^{\dagger, \S}$ and \\ Sébastien Neukirch ${ }^{\dagger}$
}

$\dagger$ †orbonne Université, Centre National de la Recherche Scientifique, UMR 7190, Institut Jean Le Rond d'Alembert, F-75005 Paris, France.

$\ddagger$ Département de Physique, École Normale Supérieure, CNRS, PSL Research University, F-75005 Paris, France.

9Matière et Systèmes Complexes, CNRS UMR 7057, Université Paris Diderot, Sorbonne Paris Cité University, F-75013 Paris, France

§Surface du Verre et Interfaces, UMR 125 CNRS/Saint-Gobain, F-93303 Aubervilliers, France.

E-mail: aurelie.fargette@dalembert.upmc.fr

\begin{abstract}
Silicone elastomers such as polydimethylsiloxane (PDMS) are convenient materials routinely used in laboratories that combine ease of preparation, flexibility, transparency and gas permeability. However, these elastomers are known to contain a small fraction of uncrosslinked low-molecular-weight oligomers, the effects of which are not completely understood, particularly when used in contact with liquids. Here we show that triple lines involving air, water, and PDMS elastomer are responsible for the contamination of water-air interfaces by uncrosslinked silicone oligomers through a capillarity-induced extraction mechanism. We investigate both the case of static and moving contact lines,
\end{abstract}


and study various geometries ranging from partially immersed PDMS plates to water droplets or air bubbles deposited on PDMS plates, all involving air-water-elastomer triple lines. We demonstrate experimentally that the contamination timescale is strikingly shorter for moving contact lines than in the static case. Eventually we propose a simple poroelastic model capturing the main features of contamination observed in experiments.

\section{Introduction}

The understanding of interactions between silicone elastomers or gels and aqueous liquids is of crucial interest in all situations where these two ingredients come into play, from elastocapillarity experiments ${ }^{1}$ to lab-on-a-chip studies, ${ }^{2}$ with applications in physics, chemistry, biology, materials science and bioengineering. Among silicone elastomers, polydimethylsiloxane (PDMS) is a popular and easy-to-use material for rapid prototyping, as it is patternable by soft lithography, optically transparent, gas-permeable, and flexible. ${ }^{3}$ This material is thus a good candidate to build microchannels for numerous applications such as vesicles or bubbles production and manipulation, ${ }^{4}$ cell cultures and organs-on-a-chip, ${ }^{5}$ or biomedical diagnostics. ${ }^{6}$ Silicone elastomers have nevertheless some drawbacks: leaching of uncrosslinked oligomers from the polymer network into microchannel media has been reported in the context of cell culture in PDMS devices. ${ }^{7,8}$ Commercial silicone elastomers such as Dow Corning Sylgard 184 PDMS indeed consist of a crosslinked polymer network but also contain a small fraction of silicone uncrosslinked chains that are not incorporated into the network during the crosslinking process. ${ }^{9}$ Beyond the recent interest for capillarity on soft solids, ${ }^{10,11}$ a better awareness of potential interactions between liquids and silicone elastomers related to capillary effects is essential to avoid or control contamination of liquid interfaces by uncrosslinked oligomers.

Consequences of the presence of uncrosslinked chains in silicone elastomers have been recently investigated in the context of adhesion, ${ }^{12}$ osmocapillary phase-separation, ${ }^{13}$ super- 
hydrophobic drag reduction, ${ }^{14}$ swelling of composite structures, ${ }^{15}$ and droplet dynamics, ${ }^{16}$ revealing that the existence of uncrosslinked chains at the scale of the polymer network can lead to significant macroscopic effects. When a silica microbead is deposited on a PDMS gel, adhesion leads to a phase separation: ${ }^{12}$ some liquid PDMS is extracted from the core of the gel at the contact line, replacing the usual three-phases contact line by a four-phases contact zone with air, silica, liquid PDMS, and gel PDMS. The question of the extraction of uncrosslinked chains is also raised when a small cavity exists at the surface of a gel, involving a competition between osmosis and capillarity. ${ }^{13}$ Additionally, traces of surfactants, possibly due to PDMS uncrosslinked chains, have been shown to severely limit the drag reduction of superhydrophobic surfaces. ${ }^{14}$ Although the presence of uncrosslinked chains is often seen as a drawback, taking advantage of uncrosslinked oligomers in silicone elastomers may also lead to functionality, shaping composite structures to form 3D structures from 2D patterns via residual swelling. ${ }^{15}$

In a previous work, ${ }^{16}$ we have shown that a water (or water-glycerol mixture) droplet deposited on an inclined plane made of silicone elastomer that contains uncrosslinked chains exhibits unusual dynamics, with two successive speed regimes, as shown in Fig. 1a. This result could not be explained by mechanisms previously reported on droplet dynamics on solid substrates. ${ }^{17,18}$ The existence of the second speed regime of a droplet on a silicone elastomer was directly linked to the presence of uncrosslinked chains in the elastomer as (i) the unusual behavior disappeared when treating the elastomer to remove the uncrosslinked chains, and (ii) an oil film was clearly visible at the surface of a beaker in which thousands of droplets were collected. Nevertheless, the exact process by which contamination of the drop surface takes place, its speed and locus remained unclear. Moreover, an alternative continuous indicator (apart from the sliding speed) of whether or not a drop is contaminated by uncrosslinked oil chains was missing.

Here we show that contact lines, whether static or in motion, mediate the contamination of droplets, and we propose that this contamination is promoted by a poroelastic capillarity- 
induced extraction of oligomers at the contact line. We first demonstrate that the extraction of uncrosslinked chains observed in the case of a droplet moving on a silicone elastomer inclined plane ${ }^{16}$ also exists in various situations where sessile or moving air-water-silicone elastomer contact lines are present (eg air bubble rising up on an immersed PDMS inclined plane). Studies of oil thin films on water baths have been performed more than one century ago for natural oils, ${ }^{19-21}$ and more recently for PDMS oils, ${ }^{22}$ showing that surface tension sharply decreases once a critical surface concentration of oil chains is reached. Building on tensiometry and ellipsometry results from Lee et al, ${ }^{22}$ we conjecture that the sharp transition in speed of droplets and bubbles is linked to the transition from patches of PDMS to a complete layer of PDMS on the water-air interface, the transition occurring once a critical surface concentration in uncrosslinked chains is reached. By performing surface tension measurements on a single droplet stopped at various positions during its descent, we indeed demonstrate that the transition in speed occurs exactly at the same time as the transition in surface tension. Depending on the situation observed (moving or sessile interfaces), either of the two parameters (speed and surface tension) is then used to quantify contamination of the water-air interface. We further establish the critical role of the presence of a water-air-PDMS contact line by comparing two simple situations: a PDMS sample completely immersed in water and a PDMS sample partially immersed in water. We evidence that the two setups lead to completely different results in terms of contamination of water-air interfaces, showing that contamination is mainly mediated by the contact line. We then study quantitatively the timescales involved in contamination of water-air interfaces in the case of sessile and moving water-air-PDMS contact lines. Eventually, we put forward an elementary model explaining contamination as a poroelastic oligomer extraction process driven by capillarity, recovering the salient features of contamination observed experimentally. 


\section{Experimental methods}

\section{Elastomers}

Elastomer samples were made of PDMS (Dow Corning Sylgard 184 Elastomer base blended with its curing agent in proportion 10:1 by weight, cured during 2 hours at $60^{\circ} \mathrm{C}$ ) molded in $12 \mathrm{~cm}$ square Petri dishes to obtain flat samples with a thickness of a few millimeters. The surface used in all droplet deposition experiments was the top surface, cured in contact with air. The Young's modulus of our PDMS samples, measured with a Shimadzu testing machine, was found to be $E=1.8 \pm 0.1 \mathrm{MPa}$.

\section{Extraction of uncrosslinked chains}

Control experiments involve PDMS samples treated with toluene to remove uncrosslinked chains. ${ }^{16}$ The extraction procedure was the following: (i) samples were immersed during one week in toluene (with no continuous agitation), the toluene bath being changed everyday to avoid saturation in uncrosslinked chains, (ii) samples were then de-swollen by progressively replacing toluene by a poor solvent of PDMS, namely ethanol, (iii) samples were dried out in a vacuum oven. Contact angles on toluene-treated samples are similar to non-treated samples (a difference in advancing and receding angles of less than $10^{\circ}$ was noticed between treated and non-treated samples).

\section{Liquids}

Droplets of deionized water (of volume comprised between 5 and $75 \mu \mathrm{L}$ ) were deposited on polymer samples with an electronic micropipette (Sartorius eLINE 5-120 $\mu \mathrm{L}$ ), with the lowest deposition speed available. 


\section{Rapid imaging acquisition and analysis}

Videos have been captured with a Hamamatsu Orca Flash digital CMOS camera, with frame rates going from 10 to 1000 frames per second. Image acquisition has been performed with ImageJ software.

\section{Surface tension measurements}

To record surface tension as a function of time, or to measure surface tension of a single droplet, Kibron EZ-Pi+ or MTX tensiometers were used with a $500 \mu$ m-diameter cylindrical platinum probe, cleaned before each measurement with a butane torch. Measuring surface tension of a small droplet with this probe however requires to take into account the Laplace pressure due to the curvature of the interface: the difference between surface tension measured in the case of a droplet and the surface tension of a flat bath of the same liquid evolves as a function of the radius of curvature of the drop, as explained in the Supplementary Information and shown in Fig. S1.

\section{Results and discussion}

\section{Contamination at triple lines}

\section{Moving droplets and bubbles}

A water - or water-glycerol mixture - droplet sliding down on a vertical PDMS plate exhibits two successive speed regimes, due to a contamination of the water-air interface of the droplet by silicone uncrosslinked chains. ${ }^{16}$ A typical height-versus-time diagram is shown in Fig. 1a. Even though the droplet might be progressively covered by uncrosslinked chains, the change to the second speed regime is abrupt. Based on measurements from Lee et al, ${ }^{22}$ we postulate that the second regime is attained once a complete layer of uncrosslinked chains is reached on the droplet. 
We now focus on what happens when reversing the phases, namely looking at an air bubble rising up an immersed PDMS plate. We first consider the case of a bubble sliding up a vertical solid plate with no uncrosslinked chains. The question of the final speed of such a bubble is mathematically identical to the question of the final speed of a droplet sliding down a vertical plate, ${ }^{23}$ except that in the bubble setup dissipation in the fluid phases mainly occurs in the surrounding liquid and not in the moving object. The simplest approach predicts that an air bubble should reach a final constant speed after a short transient. We check this hypothesis experimentally by carrying out two control experiments, on a PDMS plate treated with toluene to remove its uncrosslinked chains (Fig. 1 $b_{2}$ ), and on a vertical polyester plate that does not contain uncrosslinked chains (Fig. 1 $b_{3}$ ). In both cases, we find that the air bubble exhibits a single sliding regime. Now turning to bubble dynamics on a PDMS plate with uncrosslinked chains, we find two sliding regimes, as shown in Fig. $1 b_{1}$.

This result suggests that the contamination of the water-air interface is linked to the presence of a water-air-PDMS contact line rather than to the existence of a rolling motion inside the droplet, as the liquid flows are different in the bubble case than in the droplet case. To further investigate contamination at both moving and sessile contact lines, we then focus on the link between the speed transition and the properties of the liquid-air interface.

\section{Relationship between speed and surface tension}

As shown in Hourlier-Fargette et al, ${ }^{16}$ the surface tension of a sliding droplet varies between the beginning and the end of its two-regime descent on a PDMS sample. Here, to be able to record surface tension of a droplet during its descent, we design the following experiment: a deionized water droplet of volume $V=45 \mu \mathrm{L}$ is deposited on a Dow Corning Sylgard 184 PDMS inclined plane, making an angle equal to $45^{\circ}$ with the horizontal. Once the droplet has traveled a distance $d$ on the inclined plane, the plane is moved back into a horizontal position, and surface tension of the droplet is measured as a function of $d$ with a Kibron EZ-Pi+ tensiometer. In order to induce minimal disruption in the system, a new droplet is 

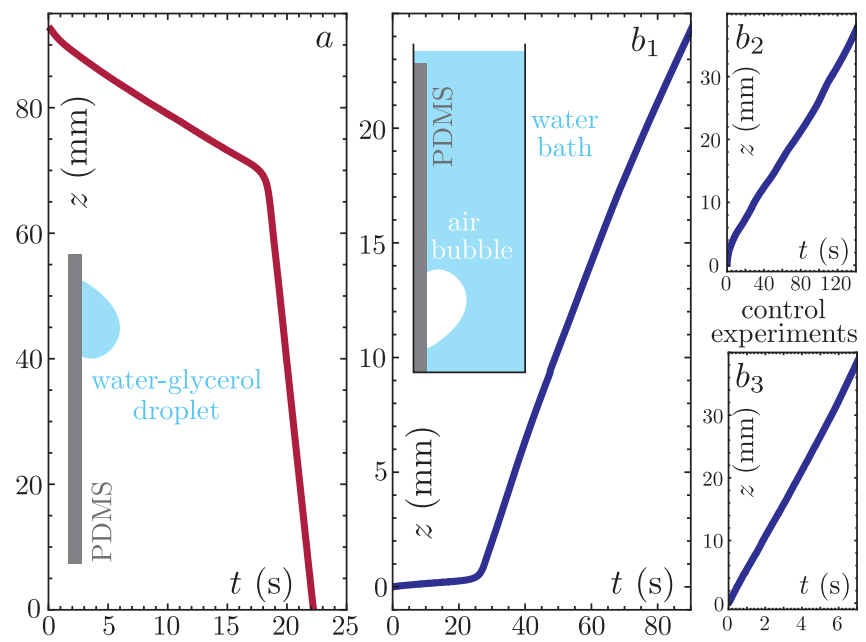

Figure 1: (a) A 40\% water - $60 \%$ glycerol mixture droplet of volume $21.5 \mu \mathrm{L}$ is deposited on a vertical PDMS plate. Its height is recorded as a function of time, and two successive speed regimes are identified. (b) A setup where phases are reversed is investigated: $\left(b_{1}\right)$ an air bubble is deposited on a vertical PDMS plate immersed in water bath and its height is recorded as a function of time. Two successive speed regimes are also identified. $\left(b_{2}\right.$ and $\left.b_{3}\right)$ As control experiments, similar air bubbles are deposited on toluene-washed PDMS (from which uncrosslinked chains have been extracted with toluene prior to the experiment, $b_{2}$ ) and on a thin polyester film $\left(b_{3}\right)$ : in both cases bubbles exhibit a single speed regime.

used for each $d$ value studied. Therefore each droplet travels a distance $d$ before its surface tension is measured within 30 seconds, and then the droplet is thrown away. The $45^{\circ}$ tilting angle is chosen to make the tilting step easier. The volume of the droplet is also chosen for its speed to be small enough to allow us to move the plane back to a horizontal position in a time lapse during which the distance traveled by the droplet is negligible compared to $d$.

Measurements of surface tension as a function of the distance $d$ traveled by the droplet are shown in Fig. 2b, where a sharp transition is observed once the droplet has traveled $8 \mathrm{~mm}$. For comparison we also show in Fig. 2a the distance-versus-time diagram for the same setup, namely a $45 \mu \mathrm{L}$ water droplet on a $45^{\circ}$ PDMS tilted plane. These results demonstrate that the speed transition occurs at the same time as a sudden surface tension transition from a value equal to the surface tension of pure water to a lower, constant, value. More precisely, we show experimentally that both transitions occur at a same distance, hence at the same time. The transition time between the two regimes can thus be identified in two different 
ways: by looking at the speed of a droplet or by measuring its surface tension.

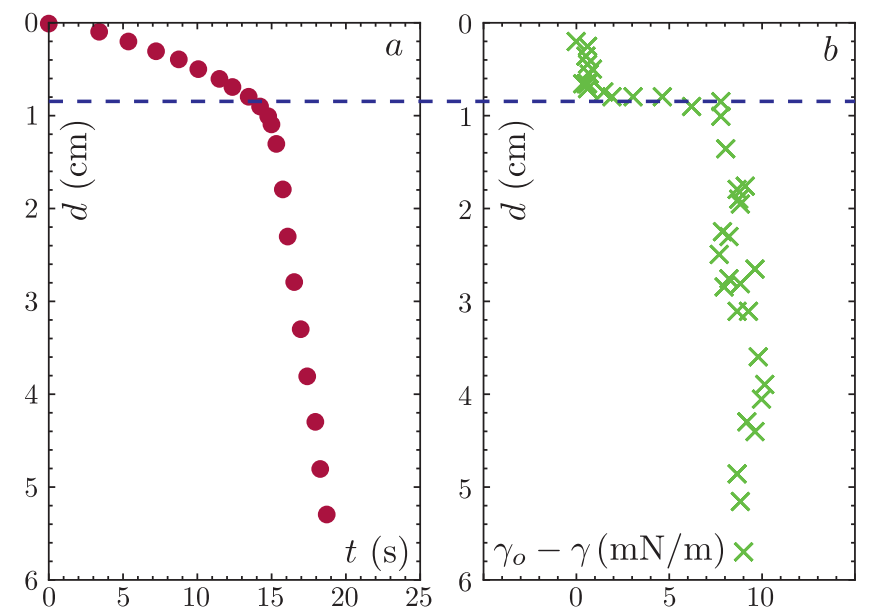

Figure 2: (a) Distance-versus-time diagram: $d$ is the distance traveled by the droplet and $t$ is time. (b) Distance-versus-surface tension diagram, $\gamma_{o}-\gamma$ is the difference between the surface tension $\gamma_{o}$ of pure water and the actual surface tension $\gamma$ of a droplet that has traveled a distance $d$. Water droplets have volume $V=45 \mu \mathrm{L}$ and are deposited on a $45^{\circ}$ inclined plane made of Dow Corning Sylgard 184 PDMS. Speed transition coincides with surface tension transition: during the first regime the droplet has a surface tension equal to the surface tension of pure water, while during the second regime its surface tension takes on another, lower, surface tension value.

\section{Sessile droplets}

We now consider contamination of water-air interfaces in the case of sessile contact lines and we use surface tension as a parameter to quantify the evolution of the contamination of the droplet as a function of time. The experiments performed in the previous section establish that quantifying the contamination timescale can be done in an equivalent manner by using either of the two parameters studied, namely speed and surface tension. Fig. 3 shows surface tension of a sessile droplet as a function of time. A sharp decrease is observed after a few minutes. This sharp transition is used to define the contamination time $\tau_{c}$ as the time for which the surface tension takes a mean value between its initial and final values. Contamination timescales for sessile droplets are much larger than contamination timescales observed for moving droplets, and are investigated further in the section entitled Contami- 
nation timescales, which includes the study of the influence of droplet size on contamination timescales, as well as a simple poroelastic modeling of those experimental results.

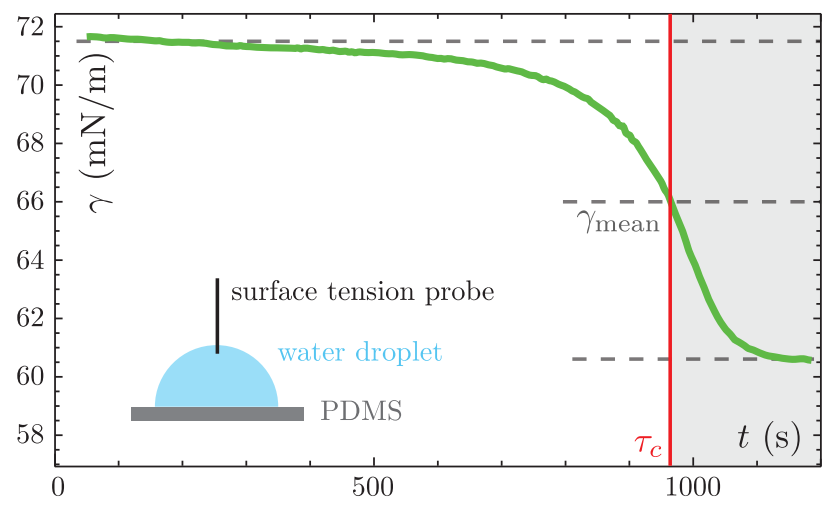

Figure 3: A $15 \mu \mathrm{L}$ deionized water droplet is deposited on a horizontal Dow Corning Sylgard 184 PDMS plate. Its surface tension is recorded as a function of time, and a neat fall-off in surface tension is observed at a time that is later referred to as $\tau_{c}$ (contamination time). More precisely, $\tau_{c}$ is defined as the time at which the average value of the initial and final surface tension, $\gamma_{\text {mean }}$, is reached. We conjecture, building on Lee et al, ${ }^{22}$ that after $\tau_{c}$ (light grey zone on the graph) a complete layer of uncrosslinked chains is formed on the droplet.

\section{Role of the triple line}

To give evidence of the crucial role of the triple line in contamination, we compare two situations where PDMS is in contact with water, with the first setup involving an water-airPDMS contact line and the second one being free of any contact line. We use two Petri dishes with diameter $5.5 \mathrm{~cm}$. In each Petri dish we lay out 5 PDMS columns, 2 with dimensions $4 \mathrm{~cm} \times 0.8 \mathrm{~cm} \times 0.5 \mathrm{~cm}$ and 3 with dimensions $2 \mathrm{~cm} \times 0.8 \mathrm{~cm} \times 0.5 \mathrm{~cm}$.

In the first Petri dish, the water level is chosen to be smaller than the height of the PDMS columns (partially immersed situation, shown in red in Fig. 4), while in the second Petri dish, the water level is a few millimeters larger than the height of the PDMS columns (totally immersed situation, shown in blue in Fig. 4). We record the surface tension of the water interface at the surface of both Petri dishes and observe that, in the case of partially immersed plates, a sharp decrease occurs around 15 minutes. In the case of totally immersed plates, we have not noticed any significant modification of the surface tension in more than 
10 hours. This experiment gives evidence that extraction is driven by the presence of a contact line.

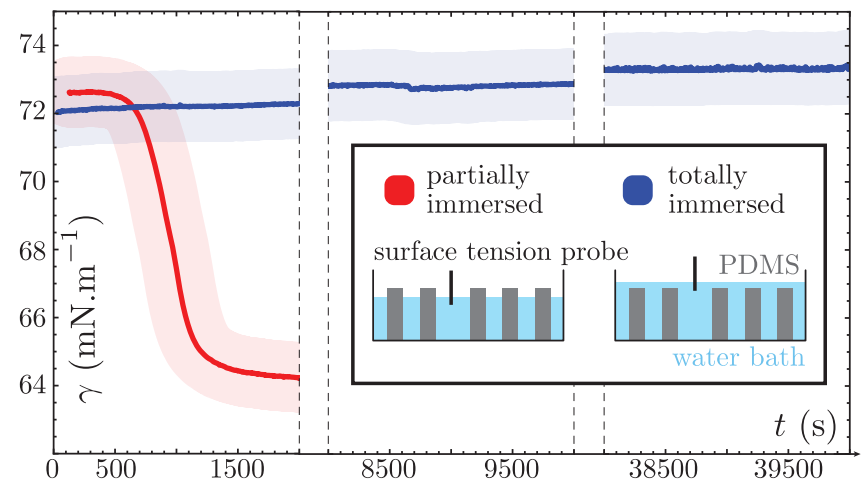

Figure 4: The surface tension of a water bath is recorded as a function of time in two situations: a first Petri dish contains partially immersed PDMS plates (red), while a second Petri dish contains completely immersed PDMS plates (blue). We observe a sharp surface tension transition for the partially immersed situation while surface tension in the totally immersed situation stays stable during the whole recording time (the small increase of the measured surface tension observed in the blue curve is due to the change in water level due to evaporation: the probe was immersed deeper than usual to avoid detachment from the liquid bath after a long recording time, and was thus subjected to Archimede's pressure). The uncertainties on the measurements are represented on the large pale colors stripes.

We further investigate the critical role of the contact line in contamination of the waterair interface by varying the immersion depth of PDMS plates with constant water-air-PDMS contact line contour-length. Two Dow Corning Sylgard 184 PDMS plates are partially immersed in a water bath, as shown in Fig. 5, with two different immersion depths. The total area of PDMS in contact with water is multiplied by a factor larger than five between the two setups: if a significant amount of PDMS chains are extracted at other locations than the triple line, the contamination time should be significantly different in the two setups. For each immersion depth, four identical experiments are performed. The results, displayed in Fig. 5, correspond to the average of the measurements for each immersion depth, error bars being representative of the statistical uncertainties. No significant difference is observed in the contamination time $\tau_{c}$ between the two setups, giving additional evidence that extraction of uncrosslinked chains essentially occurs at the triple line. 


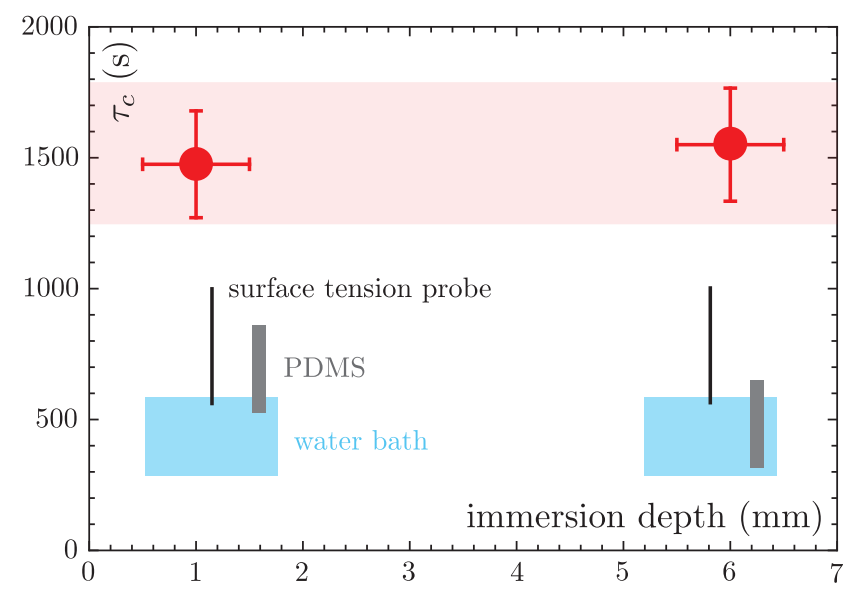

Figure 5: Dependence of the contamination time $\tau_{c}$ as function of the immersion depth. Dow Corning Sylgard 184 plates are partially immersed in a water bath of total area $4 \mathrm{~cm}^{2}$. The perimeter of the contact line between PDMS and water is $36 \mathrm{~mm}$. Each point on the graph is an average of four identical measurements, and the error bars correspond to statistical uncertainties. An increase of the immersion depth by more than a factor five leads to no significant modification of the contamination time $\tau_{c}$. The light red band is a guide for the eye and illustrates that, given the uncertainties, the contamination times in the two cases can be considered to be equal.

\section{Contamination timescales}

\section{Sessile droplets}

We now focus on contamination timescales, and investigate first the contamination dynamics of a sessile droplet as a function of the droplet size. Our experimental setup is the following: a droplet of volume $V$ is deposited on a horizontal Dow Corning Sylgard 184 PDMS plate, and its surface tension is recorded as a function of time. The contamination time $\tau_{c}$ is determined for each droplet volume by taking the average of one to three measurements, and the error bars are representative of statistical uncertainties. Volumes ranging from $5 \mu \mathrm{L}$ to $75 \mu \mathrm{L}$ are investigated. The contamination time $\tau_{c}$ is then plotted in Fig. 6 as a function of the base radius of the droplet $R$, calculated from the droplet volume $V$ by assuming that the droplet is a half sphere. A first conclusion is that the timescales recorded for sessile droplets contamination are much longer than typical timescales involved in the case of moving droplets or bubbles. We also observe that the experimental results exhibit a rather 
linear relationship between the contamination time $\tau_{c}$ and the base radius $R$, relationship that will be analyzed further in the next section.

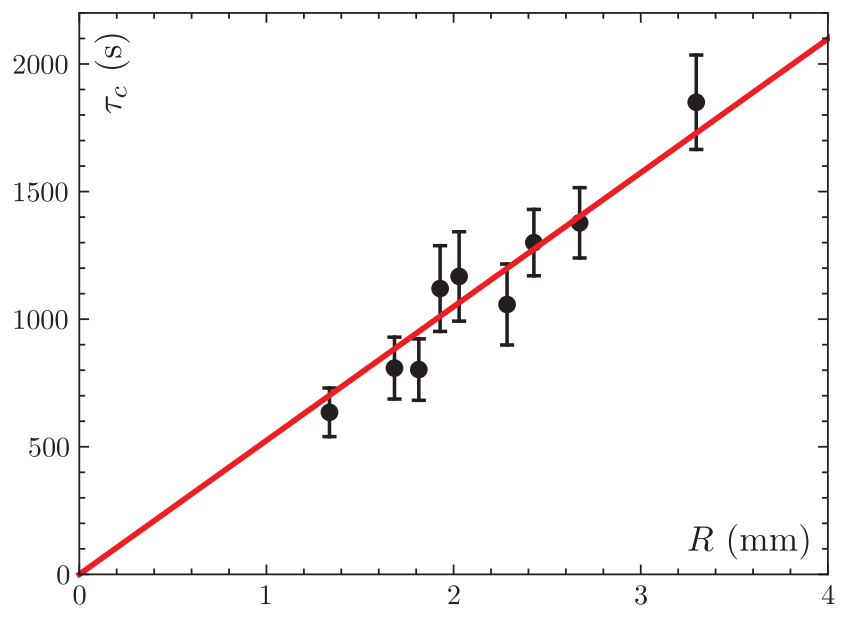

Figure 6: Contamination time $\tau_{c}$ as a function of the base radius $R$ of a sessile water droplet deposited on a Dow Corning Sylgard 184 plate. Each point is an average of one to three measurements, the error bars being representative of statistical uncertainties. The red line is a linear fit, showing that our results are compatible with a linear relation $\tau_{c} \propto R$.

\section{Moving droplets}

Contamination of moving droplets involve much shorter timescales than sessile droplets. However, at fixed droplet size, the contamination timescale - defined as the time at which the second speed regime is reached - depends on the speed of the droplet. To quantify the evolution of the contamination timescale as a function of the droplet speed, our experimental setup is the following: water droplets of constant volume are deposited on PDMS inclined planes, and the angle of inclination is varied in order to change the droplet speed in the first regime without affecting its volume. Changing the inclination angle instead of the droplet volume allows us to reach different speeds without varying significantly the perimeter of the contact line (which is a parameter that is likely to influence the contamination time given results obtained for sessile droplets). Experiments are performed with three droplet volumes, to rapidly test the influence of the droplet size. The contamination time is recorded as a function of the speed of the droplet and shown in a log-log diagram in Fig 7 . Yellow 
triangles correspond to $20 \mu \mathrm{L}$ droplets, orange dots to $40 \mu \mathrm{L}$ droplets, and red squares to $60 \mu \mathrm{L}$ droplets. Angles of inclination are varied between $57^{\circ}$ and $90^{\circ}$ for $20 \mu \mathrm{L}$ droplets, between $33^{\circ}$ and $45^{\circ}$ for $40 \mu \mathrm{L}$ droplets, and between $24^{\circ}$ and $35^{\circ}$ for $60 \mu \mathrm{L}$ droplets. The droplets shapes are slightly different when changing the angle of the inclined plane, but to first order, this shape modification will be neglected in the discussion. No significant difference is observed between the curves for the different volumes. This can be explained by the limited volume variation range accessible in our experiments: droplets of smaller volumes are immobile on a vertical plane due to pinning forces, while droplets of larger volume form puddles. Uncertainties, that are mainly statistical and can be estimated through the scatter of our data, do not allow us to distinguish between the three volume cases. A power law with a negative exponent (lower than unity in absolute value, close to $-2 / 3$ ) fits well the data, except from a small deviation at small speeds. This deviation was to be expected, as the contamination time in the case of a sessile droplet is not infinite. Further analysis of the experimental scaling law established here is performed in the following section.

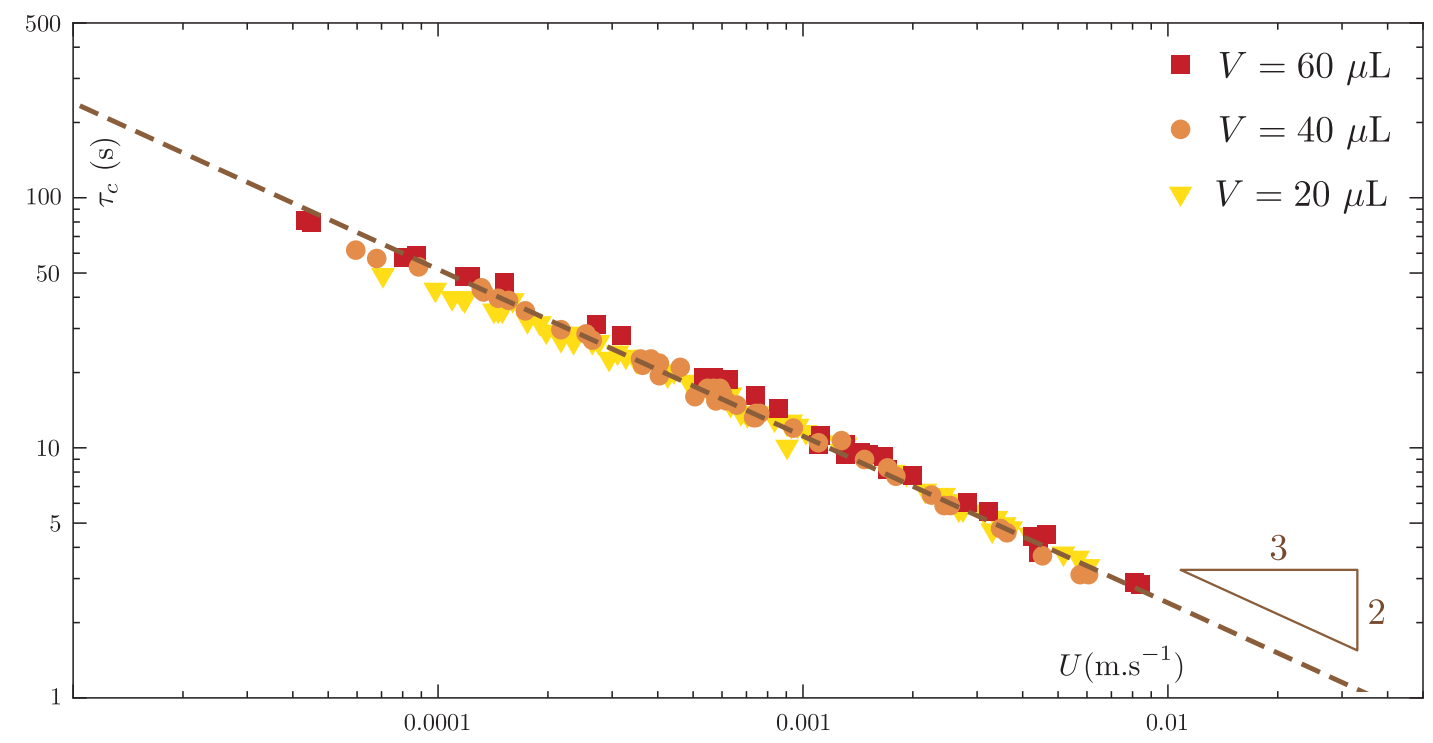

Figure 7: Water droplets of volumes $20 \mu \mathrm{L}$ (yellow triangles), $40 \mu \mathrm{L}$ (orange dots), and $60 \mu \mathrm{L}$ (red squares) are deposited on Dow Corning Sylgard 184 PDMS inclined planes. The angle of inclination is varied to change the droplet speed. We record the contamination time $\tau_{c}$ as a function of the droplet speed in the first regime $U$, and show that our data are consistent with a $-2 / 3$ power law scaling (brown dashed line). 


\section{Contamination timescales: a scaling approach}

In a poroelastic material, solvent molecules (free chains in the case of PDMS) move in response to gradients of chemical potential (also known as the "pore pressure") which depends on both the local solvent concentration and the state of stress of the material. ${ }^{24}$ At the triple line between an elastic solid, a vapour phase and a liquid phase, capillarity leads to the formation of a sharp ridge beneath the contact line where the elastic material is under tension. ${ }^{25,26}$ The characteristic length of this ridge is given by the elastocapillary length ${ }^{27}$ $\ell_{s} \sim \gamma_{s} / E$ where $\gamma_{s}$ and $E$ are respectively the surface tension and the elastic modulus of the PDMS. As theoretically suggested recently, ${ }^{27}$ this tension may induce the migration of free chains and their accumulation within the ridge. This diffusive process occurs on a typical timescale $t_{\text {diff }}=\ell_{s}^{2} / D$ where $D$ is the effective diffusion coefficient of the free chains in the crosslinked network, estimated to be in the range $10^{-12}-10^{-11} \mathrm{~m}^{2} / \mathrm{s}^{27,28} \mathrm{In}$ our system $\ell_{s}$ is around $10 \mathrm{~nm}$ and the timescale $t_{\text {diff }}$ is thus of the order of $10 \mu \mathrm{s}$. Because it is energetically favorable for PDMS chains to cover the surface of the water droplet, there is a flux of chains per unit area $J$ from the concentrated ridge towards the surface of the drop.

At small time $\left(t \ll t_{\text {diff }}\right.$ ), this flux diverges as $\sim 1 / \sqrt{t}$, while at $t \rightarrow \infty$ it vanishes as the chemical potentials of the PDMS chains in the elastomer and at the surface of the drop will be equal. ${ }^{29}$ However, if we assume that the diffusion of the chains at the surface of the drop is significantly faster ${ }^{30}$ than their diffusion throughout the elastomer/droplet interface, then while the concentration of chains is much higher in the PDMS ridge (which acts as an infinite reservoir of free chains) than at the surface of the drop, the flux $J$ will be constant and essentially proportional to the free chains concentration in the elastomer. We assume that the diffusive process of the chains through the triple line is the limiting process, and will check the consistency of this hypothesis shortly thereafter. 


\section{Model for sessile droplets}

The total flux $J_{\text {tot }}$ of small molecules from the PDMS ridge toward the surface of the drop can be estimated as the flux $J$ multiplied by an area. As mentioned previously, uncrosslinked chains are essentially concentrated in the elastocapillary ridge which, in three dimensions, is a ring of length proportional to $R$ and of thickness $\ell_{s}$. The total flux from the PDMS to the drop surface is therefore simply given by $J_{\text {tot }} \sim J R \ell_{s}$ and thus behaves as $J_{\text {tot, small } t} \propto R \ell_{s} / \sqrt{t}$ at small times and as $J_{\text {tot, large } t} \propto R \ell_{s}$ at large times. Now, the total number of free chains $N(t)$ at the free surface of the drop at time $t$ is given by the integral $N(t)=\int_{0}^{t} J_{\text {tot }}(t) \mathrm{d} t$. Furthermore, there exists a critical number $N_{c}$ of free chains at the free surface of the drop that triggers the surface tension transition. This number is proportional to the area of the drop, $N_{c} \propto \rho_{c} R^{2}$ where $\rho_{c}$ is the critical surface concentration of free chains. As a consequence, the transition occurs at the critical time $\tau_{c}$ when the total number of extracted molecules $N\left(\tau_{c}\right)$ reaches the critical number $N_{c}$ i.e:

$$
N\left(\tau_{c}\right)=\int_{0}^{\tau_{c}} J_{\mathrm{tot}} \mathrm{d} t=N_{c} \propto \rho_{c} R^{2}
$$

For large sessile drops, the timescale of contamination is much longer than the diffusive

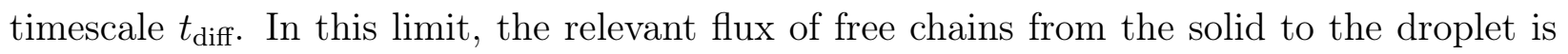
thus the large times flux $J_{\text {tot, large } t} \propto R \ell_{s}$. Inserting this expression in the equation above, one obtains a critical contamination time:

$$
\tau_{c, \text { sessile drop }} \propto \frac{\rho_{c} R}{\ell_{s}}
$$

showing that the contamination time is indeed proportional to the base radius $R$ of the drop and thus in good agreement with the results of Fig. 6 . 


\section{Model for moving droplets}

If now the drop is in motion, the typical width of the ring acting as a reservoir of uncrosslinked chains is no longer the elastocapillary length $\ell_{s}$, but the length scanned during the motion of the drop, i.e $U t$ where $U$ is the velocity of the drop. The total flux from the solid to the drop surface is simply $J_{\text {tot }} \propto J R U t$ and thus behaves as $J_{\text {tot, small } t} \propto R U t / \sqrt{t}$ at small times and as $J_{\text {tot, large } t} \propto R U t$ at large times. If the drop moves fast enough (i.e $U \ll \ell_{s} / t_{\text {diff }} \sim$ $10^{-4} \mathrm{~m} / \mathrm{s}$ ), as it is the case in our experimental setup, the relevant approximation for the flux of uncrosslinked chains is thus $J_{\text {tot, small } t} \propto R U t / \sqrt{t}$. Inserting this expression in (1) leads to the critical contamination time:

$$
\tau_{c, \text { moving drop }} \propto\left(\frac{\rho_{c} R}{U}\right)^{2 / 3}
$$

where we recover the scaling $U^{-2 / 3}$ observed in the experiments shown in Fig 7 . As $U \rightarrow 0$, the critical time $\tau_{c}$ is not diverging, as observed in Fig. 6. Consequently the scaling $U^{-2 / 3}$ is eventually lost at small speeds for which we expect a quantitatively different behavior, with a weaker influence of the velocity on the contamination time. Hence, the present simple model is unlikely to predict contamination timescales at low to intermediate velocities because it would be necessary to take into account the full time-dependent evolution of the concentration field in the ridge, which is outside the scope of this study.

In addition, we estimate the diffusion coefficient of the PDMS chains across the triple line. From the slope of the curve $\tau_{c}(R)$ shown in Fig 6, as well as an estimation of the critical surface concentration, $\rho_{c} \sim 0.7 \mathrm{mg} / \mathrm{m}^{2},{ }^{22}$ we evaluate the linear mass flow rate from the PDMS to the drop as $Q=\rho_{c} R / \tau_{c} \sim 10^{-6} \mathrm{mg} / \mathrm{m} / \mathrm{s}$. As mentioned previously, we have assumed that this rate is directly proportional to the concentration of free chains in the ridge. While this concentration is not known, we may broadly estimate its value to lie between the typical concentration of free chains in a PDMS elastomer $(\sim 5 \% 9,16)$ and that of a fully liquified ridge (100\%). Because the volumetric mass density of PDMS is around 
$1000 \mathrm{~kg} / \mathrm{m}^{3}$, the diffusion coefficient of the chains across the interface is therefore in the range $Q / \rho \sim 10^{-15}-10^{-13} \mathrm{~m}^{2} / \mathrm{s}$. This value is well below the diffusion coefficient of PDMS chains within the crosslinked network ${ }^{27}$ and thus consistent with our earlier hypothesis that the limiting process for the droplet contamination is the crossing of the elastomer surface by the PDMS chains.

\section{Conclusion}

We have shown that silicone uncrosslinked chains present in most commonly used silicone elastomers contaminate air-water interfaces when a air-water-silicone elastomer contact line is present. By performing experiments in several configurations, with droplets, bubbles, and water baths, we have demonstrated that the contact line does not need to move in order to promote interfacial contamination: modifications of the air-water interfacial properties have been highlighted both in the case of moving and sessile contact lines. Contamination timescales have been shown to differ from several orders of magnitude between sessile and moving contact lines. More precisely, the migration of silicone uncrosslinked chains to the interface leads to a sharp surface tension transition once a critical surface concentration in chains is reached. This transition is defined as the contamination timescale. In the context of moving contact lines, we have found that the surface tension transition occurs exactly at the moment when a sharp speed transition is observed, which makes this transition visually striking. For sessile contact lines, surface tension was recorded to determine the contamination timescale. The use of those two physical parameters allowed us to collect measurements both for sessile and moving contact lines, that have been successfully compared to a simple model using a diffusion driven mechanism for the migration of the chains.

Soft materials and especially silicone elastomers are widely used in contact with water in many research fields. However, interactions between silicone elastomers and liquids are often forgotten, but can lead to unexpected behaviors that can be explained in the light of 
the present work.

\section{Acknowledgement}

We thank Emmanuelle Rio and Vivek Sharma for their support in the experiments involving Kibron tensiometers, Antoine Chateauminois for his help in the preparation of toluenewashed elastomer samples for control experiments, and Paul Grandgeorge for enlightening discussions. The present work was supported by ANR grant ANR-14-CE07-0023-01.

\section{Supporting Information Available}

Supplementary information includes a 3 pages pdf document and one figure S1, describing the experimental technique used to measure the surface tension of a single droplet, taking into account Laplace pressure.

This material is available free of charge via the Internet at http://pubs.acs.org/.

\section{References}

(1) Bico, J.; Reyssat, E.; Roman, B. Elastocapillarity: When Surface Tension Deforms Elastic Solids. Annual Review of Fluid Mechanics 2018, 50, 629-659.

(2) Whitesides, G. M. The origins and the future of microfluidics. Nature 2006, 442, 368373.

(3) Mukhopadhyay, R. When PDMS isn't the best. Analytical Chemistry 2007, 79, 32483253.

(4) Gañán Calvo, A. M.; Gordillo, J. M. Perfectly Monodisperse Microbubbling by Capillary Flow Focusing. Phys. Rev. Lett. 2001, 87, 274501. 
(5) Huh, D.; Torisawa, Y.; Hamilton, G. A.; Kim, H. J.; Ingber, D. E. Microengineered physiological biomimicry: Organs-on-Chips. Lab. Chip 2012, 12, 2156-2164.

(6) Chin, C. D. et al. Microfluidics-based diagnostics of infectious diseases in the developing world. Nat. Med. 2011, 17, 1015-1019.

(7) Berthier, E.; Young, E. W. K.; Beebe, D. Engineers are from PDMS-land, Biologists are from Polystyrenia. Lab. Chip 2012, 12, 1224-1237.

(8) Regehr, K. J.; Domenech, M.; Koepsel, J. T.; Carver, K. C.; Ellison-Zelski, S. J.; Murphy, W. L.; Schuler, L. A.; Alarid, E. T.; Beebe, D. J. Biological implications of polydimethylsiloxane-based microfluidic cell culture. Lab. Chip 2009, 9, 2132-2139.

(9) Lee, J. N.; Park, C.; Whitesides, G. M. Solvent Compatibility of Poly(dimethylsiloxane)-Based Microfluidic Devices. Analytical Chemistry 2003, $75,6544-6554$.

(10) Andreotti, B.; Baumchen, O.; Boulogne, F.; Daniels, K. E.; Dufresne, E. R.; Perrin, H.; Salez, T.; Snoeijer, J. H.; Style, R. W. Solid capillarity: when and how does surface tension deform soft solids? Soft Matter 2016, 12, 2993-2996.

(11) Style, R. W.; Jagota, A.; Hui, C.-Y.; Dufresne, E. R. Elastocapillarity: Surface Tension and the Mechanics of Soft Solids. Annual Review of Condensed Matter Physics 2017, 8, 99-118.

(12) Jensen, K. E.; Sarfati, R.; Style, R. W.; Boltyanskiy, R.; Chakrabarti, A.; Chaudhury, M. K.; Dufresne, E. R. Wetting and phase separation in soft adhesion. Proceedings of the National Academy of Sciences 2015, 112, 14490-14494.

(13) Liu, Q.; Suo, Z. Osmocapillary phase separation. Extreme Mechanics Letters 2016, 7, $27-33$. 
(14) Peaudecerf, F. J.; Landel, J. R.; Goldstein, R. E.; Luzzatto-Fegiz, P. Traces of surfactants can severely limit the drag reduction of superhydrophobic surfaces. Proceedings of the National Academy of Sciences 2017, 114, 7254-7259.

(15) Pezzulla, M.; Shillig, S. A.; Nardinocchi, P.; Holmes, D. P. Morphing of geometric composites via residual swelling. Soft Matter 2015, 11, 5812-5820.

(16) Hourlier-Fargette, A.; Antkowiak, A.; Chateauminois, A.; Neukirch, S. Role of uncrosslinked chains in droplets dynamics on silicone elastomers. Soft Matter 2017, 13, $3484-3491$.

(17) Le Grand, N.; Daerr, A.; Limat, L. Shape and motion of drops sliding down an inclined plane. J. Fluid Mech. 2005, 541, 293-315.

(18) Puthenveettil, B. A.; Senthilkumar, V. K.; Hopfinger, E. J. Motion of drops on inclined surfaces in the inertial regime. Journal of Fluid Mechanics 2013, 726, 26-61.

(19) Pockels, A. On the Relative Contamination of the Water-Surface by Equal Quantities of Different Substances. Nature 1892, 46, 418-419.

(20) Rayleigh, L. XXXVI. Investigations in Capillarity: The size of drops. The liberation of gas from supersaturated solutions. Colliding jets. The tension of contaminated watersurfaces. Philosophical Magazine Series 5 1899, 48, 321-337.

(21) Langmuir, I. The constitution and fundamental properties of solids and liquids. II. Liquids. Journal of the American Chemical Society 1917, 39, 1848-1906.

(22) Lee, L. T.; Mann, E. K.; Langevin, D.; Farnoux, B. Neutron reflectivity and ellipsometry studies of a polymer molecular layer spread on the water surface. Langmuir 1991, 7 , 3076-3080.

(23) Dussan V., E. B.; Chow, R. T.-P. On the ability of drops or bubbles to stick to nonhorizontal surfaces of solids. Journal of Fluid Mechanics 1983, 137, 1-29. 
(24) Biot, M. A. General theory of three-dimensional consolidation. Journal of applied physics 1941, 12, 155-164.

(25) Shanahan, M.; Carré, A. Spreading and dynamics of liquid drops involving nanometric deformations on soft substrates. Colloids and Surfaces A: Physicochemical and Engineering Aspects 2002, 206, 115 - 123 .

(26) Park, S. J.; Weon, B. M.; San Lee, J.; Lee, J.; Kim, J.; Je, J. H. Visualization of asymmetric wetting ridges on soft solids with X-ray microscopy. Nature Communication 2014, 5, 4369.

(27) Zhao, M.; Lequeux, F.; Narita, T.; Roché, M.; Limat, L.; Dervaux, J. Growth and relaxation of a ridge on a soft poroelastic substrate. Soft Matter 2018, 14, 61-72.

(28) Garrido, L.; Mark, J. E.; Ackerman, J. L.; Kinsey, R. A. Studies of self-diffusion of poly (dimethylsiloxane) chains in PDMS model networks by pulsed field gradient NMR. Journal of Polymer Science Part B: Polymer Physics 1988, 26, 2367-2377.

(29) Carslaw, H. S.; Jaeger, J. C. Conduction of heat in solids; Oxford: Clarendon Press, 2nd ed., 1959.

(30) Bergeron, V.; Langevin, D. Monolayer Spreading of Polydimethylsiloxane Oil on Surfactant Solutions. Phys. Rev. Lett. 1996, 76, 3152-3155. 


\section{Graphical TOC Entry}

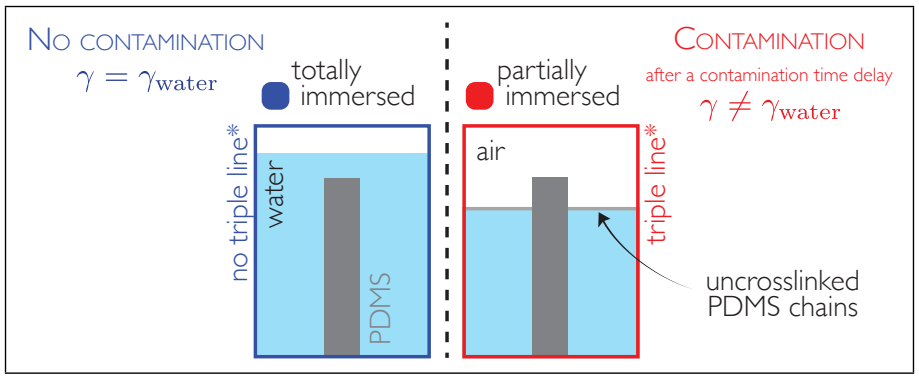

